\title{
MHD Boundary Layer Flow of Nanofluid over a Continuously Moving Stretching Surface
}

\author{
Haroon Rasheed, Abdul Rehman", Naveed Sheikh, Saleem Iqbal \\ Department of Mathematics, University of Balochistan, Quetta, Pakistan \\ Email address: \\ abdul_maths@yahoo.com (A. Rehman) \\ ${ }^{*}$ Corresponding author \\ To cite this article: \\ Haroon Rasheed, Abdul Rehman, Naveed Sheikh, Saleem Iqbal. MHD Boundary Layer Flow of Nanofluid over a Continuously Moving \\ Stretching Surface. Applied and Computational Mathematics. Vol. 6, No. 6, 2017, pp. 265-270. doi: 10.11648/j.acm.20170606.15
}

Received: October 25, 2017; Accepted: November 13, 2017; Published: December 25, 2017

\begin{abstract}
The present investigation provides an insight in the steady, incompressible and electrically conducting boundary layer flow of viscoelastic nanofluid flowing due to a moving, linearly stretched surface. The governing system of nonlinear partial differential equations is simplified by considering Boussinesq and boundary layer approximations. An analytical solution of the resulting nonlinear ordinary differential equations for momentum, energy and concentration profiles is obtained using the homotopy analysis method (HAM).
\end{abstract}

Keywords: Magnetohydrodynamic, Boundary Layer Flow, Nanoparticle, Moving Surface

\section{Introduction}

Enhancement of thermal conductivity in the base fluid due to the presence of nano sized solid particles is studied vigorously by different researchers in the recent past. The properties and behavior of base fluid are exceedingly dependent upon the solid particles size, shape and their associated dispersion features $[1,2]$. Presence of nanoparticles resembles to a significant alteration in the viscosity and the specific heat of the base fluids [3]. Khan et al. [3] have deliberated the effects of thermal radiation, heat generation and chemical readtion over the magnetohydrodynamic laminar boundary layer flow of a nanofluid past a wedge. Nadeem and Rehman [4] have presented an analytical solution for the finite radial domain, axisymmetric stagnation flow of a nanofluid flowing between the annular region formed by two concentric cylinders, when the inner cylinder is translating along and rotating about the axial direction with constant linear and angular velocities. Further, Rehman et al. [5] have debated the problem of nonNewtonian couple stress fluid containing nanoparticles flowing over a stretching surface when the stretching velocity varies exponentially with the distance from the stagnation point. The magneto-hydrodynamic boundary layer flow of a nanofluid flowing through some porous medium over an exponentially stretching surface was studied by Ferdows et al. [6]. Khan et al. [7] have analyzed the time dependent boundary layer flow of nanofluid flowing along a stretced surface and is under the influence of a magnetic field with viscous dissipation and thermal radiation. They [7] observed that the boundary layer concentration of nanoparticles is highly dependent upon their size and shape. Few other motivating studies about the flow behavior of nanofluids are cited in [8-13].

The purpose of the present study is to analyze the effects of nanoparticles over the heat transfer and viscoelastic nonNewtonian second grade, steady incompressible, electrically conducting boundary layer fluid flow due to a moving, linearly stretched sheet. The governing system of nonlinear partial differential equations and the associated boundary conditions describing the problem are first shortened by applying Boussinesq and boundary layer approximations. The resulting nonlinear system of partial differential equations is then creamed into a nonlinear syste of ordinary differential equations using a proper similarity transformation. An analytic solution of the resulting system of nonlinear ordinary differential equations for momentum, energy and concentration profiles is obtained using the 
homotopy analysis method (HAM). Convergence of the solutions and the physical behavior of the important involved parameters are discussed at the end.

\section{Formulation}

Let us consider a steady, two dimensional, incompressible

$$
\begin{gathered}
\frac{\partial u}{\partial x}+\frac{\partial v}{\partial y}=0 \\
u \frac{\partial u}{\partial x}+v \frac{\partial u}{\partial y}=-k_{0}\left[u \frac{\partial^{3} u}{\partial x \partial y^{2}}+v \frac{\partial^{3} u}{\partial y^{3}}+\frac{\partial u}{\partial x} \frac{\partial^{2} u}{\partial y^{2}}-\frac{\partial u}{\partial y} \frac{\partial^{2} u}{\partial x \partial y}\right]+v \frac{\partial^{2} u}{\partial y^{2}} \\
-\frac{\sigma B_{0}^{2}}{\rho} u+g \beta\left[\frac{1}{\rho \beta}\left(\rho^{*}-\rho\right)\left(\varphi-\varphi_{\infty}\right)+\left(1-\varphi_{\infty}\right)\left(T-T_{\infty}\right)\right] \\
u \frac{\partial T}{\partial x}+v \frac{\partial T}{\partial y}=\frac{1}{\rho c_{p}} \frac{\partial}{\partial y}\left(k \frac{\partial T}{\partial y}\right)-\frac{1}{\rho c_{p}} \frac{\partial q_{r}}{\partial y}+\frac{\rho^{*} c_{p}^{*}}{\rho c_{p}}\left(D_{B} \frac{\partial T}{\partial y} \frac{\partial \varphi}{\partial y}+\frac{D_{T}}{T_{\infty}}\left(\frac{\partial T}{\partial y}\right)^{2}\right) \\
u \frac{\partial \varphi}{\partial x}+v \frac{\partial \varphi}{\partial y}=D_{B} \frac{\partial^{2} \varphi}{\partial y^{2}}+\frac{D_{T}}{T_{\infty}} \frac{\partial^{2} T}{\partial y^{2}}
\end{gathered}
$$

where $u$ and $v$ are the velocity components along the $x$ and $y$ directions, $\rho$ is density, $v$ is the kinematic viscosity, $g$ is the gravitational acceleration, $\beta$ is the coefficient of thermal expansion, $k_{0}$ is the viscoelastic fluid parameter, $\sigma$ is the electric conductivity, $B_{0}$ is the external magnetic field acting in y direction, $T$ is the temperature, $k$ is the thermal diffusivity, $c_{p}$ is the specific heat of the viscoelastic fluid at constant pressure, $\rho^{*}$ is the nanoparticle mass density, $c_{p}^{*}$ is the effective heat of nanoparticles, $D_{B}$ is the Brownian diffusion coefficient, $D_{T}$ is the thermophoretic diffusion coefficient and $\varphi$ is the nanoparticle concentration function. The corresponding boundary conditions for the problem are

$$
\begin{gathered}
u=u_{w}(x), \quad v=0, \quad \text { at } y=0, \quad u \rightarrow 0, \quad \frac{\partial u}{\partial y} \rightarrow 0, \quad \text { as } y \rightarrow \infty, \\
T=T_{w}=T_{\infty}+A(x / l), \quad \text { at } y=0, \quad T \rightarrow T_{\infty}, \quad \text { as } y \rightarrow \infty, \\
\varphi=\varphi_{w}=\varphi_{\infty}+x / l, \text { at } y=0, \varphi \rightarrow \varphi_{\infty}, \text { as } y \rightarrow \infty .
\end{gathered}
$$

Using the stream function $\psi$ as introduced by Rashidi et al. [14] and the other similarity variables

$$
\eta=\sqrt{\frac{u_{w}(x)}{v x}} y, \quad \psi=\sqrt{v x u_{w}(x)} f(\eta), \quad \theta=\frac{T-T_{\infty}}{T_{w}-T_{\infty}}, \quad \Psi=\frac{\varphi-\varphi_{\infty}}{\varphi_{w}-\varphi_{\infty}},
$$

With the help of transformations in $E q$. (8), the governing boundary layer equations can be written as

$$
\begin{gathered}
f_{\eta \eta \eta}+f f_{\eta \eta}-f_{\eta}^{2}+k_{1}\left(f f_{\eta \eta \eta \eta}+f_{\eta \eta}^{2}-2 f_{\eta} f_{\eta \eta \eta}\right)-M n f_{\eta}+G r\left(1-\varphi_{\infty}\right)\left(\theta+N r^{*} \Psi\right)=0 \\
(1+\varepsilon \theta+N r) \theta_{\eta \eta}+\operatorname{Pr}\left(f \theta_{\eta}-f_{\eta} \theta\right)+\varepsilon \theta_{\eta}^{2}+N b \theta_{\eta} \Psi_{\eta}+N t \theta_{\eta}^{2}=0
\end{gathered}
$$$$
\Psi_{\eta \eta}+S c\left(f \Psi_{\eta}-f_{\eta} \Psi\right)+\frac{N t}{N b} \theta_{\eta \eta}=0
$$

in which $k_{1}=k_{0} b / v$ is the viscoelastic parameter, $M n=\sigma B_{0}^{2} / b \rho$ is the magnetic parameter, $G r=g \beta A / b^{2} l$

$$
N r^{*}=\left(\rho^{*}-\rho\right)\left(\varphi_{w}-\varphi_{\infty}\right) / \rho \beta\left(1-\varphi_{\infty}\right)\left(T_{w}-T_{\infty}\right) \quad \text { is the }
$$$$
\text { is the Grashof number, }
$$
buoyancy ratio, $\operatorname{Pr}=\mu c_{p} / K_{\infty}$ is the Prandtl number, $N r=16 \sigma^{*} T_{\infty}^{3} / 3 K^{*} K_{\infty}$ is the thermal radiation parameter, 
$N b=\rho^{*} c_{p}^{*} D_{B}\left(\varphi_{w}-\varphi_{\infty}\right) / K_{\infty}$ is the Brownian motion parameter, $N t=\rho^{*} c_{p}^{*} D_{T}\left(T_{w}-T_{\infty}\right) / T_{\infty} K_{\infty}$ is the thermophoresis parameter and $S c=v / D_{B}$ is the Schmidt number. The boundary conditions in nondimensional form can be written as

$$
\begin{gathered}
f(0)=0, \quad f_{\eta}(0)=1, \quad \theta(0)=1, \quad \Psi(0)=1, \\
f_{\eta} \rightarrow 0, \quad f_{\eta \eta} \rightarrow 0, \quad \theta \rightarrow 0, \quad \Psi \rightarrow 0, \quad \text { as } \eta \rightarrow \infty
\end{gathered}
$$

Further the problem is solved using the analytic technique the homotopy analysis method.

\section{Solution of the Problem}

To obtain the solutions of Eqs. (10) and (11) subject to the boundary conditions in Eqs. (12) and (13) by homotopy analysis method choose the initial guesses as

$$
f_{0}(\eta)=1-e^{-\eta}, \quad \theta_{0}(\eta)=e^{-\eta}, \quad \Psi_{0}(\eta)=e^{-\eta}
$$

The associated linear operators for the velocity, temperature and concentration profiles are

$$
L_{f}=\frac{d^{3}}{d \eta^{3}}+\frac{d^{2}}{d \eta^{2}}, \quad L_{\theta}=\frac{d^{2}}{d \eta^{2}}+\frac{d}{d \eta}, \quad L_{\Psi}=\frac{d^{2}}{d \eta^{2}}+\frac{d}{d \eta}
$$

These linear operators satisfy the conditions

$$
\begin{aligned}
& L_{f}\left[c_{1}+c_{2} \eta+c_{3} e^{-\eta}\right]=0, \\
& L_{\theta}\left[c_{4}+c_{5} e^{-\eta}\right]=0, \\
& L_{\Psi}\left[c_{6}+c_{7} e^{-\eta}\right]=0,
\end{aligned}
$$

where $c_{i}(i=1,2, \ldots, 7)$ are arbitrary constants. The zerothorder deformation equations are

$$
\begin{aligned}
& (1-q) L_{f}\left[\hat{f}(\eta ; q)-f_{0}(\eta)\right]=q \hbar_{1} N_{f}[\hat{f}(\eta ; q)], \\
& (1-q) L_{\theta}\left[\hat{\theta}(\eta ; q)-\theta_{0}(\eta)\right]=q \hbar_{2} N_{\theta}[\hat{\theta}(\eta ; q)], \\
& (1-q) L_{\Psi}\left[\hat{\Psi}(\eta ; q)-\Psi_{0}(\eta)\right]=q \hbar_{3} N_{\Psi}[\hat{\Psi}(\eta ; q)],
\end{aligned}
$$

Further details about the HAM procedure can be found in [14-26].

\section{Convergence of the HAM Solutions}

Figures (1), (2) and (3) are comprised to survey the convergence of the homotopy solutions for the velocity, temperature and concentration profiles. Figure (1) exhibits the boundary derivatives corresponding to the convergence arcs for the nondimensional velocity profiles $f^{\prime}$ schemed against the different values of the magnetic parameter $M n$ plotted for the $20^{\text {th }}$ order approximation of the HAM solutions, considering the other parameters fixed at the $0.02 \%$ level of the nanoparticle concentration inside the second grade base fluid. Figure (1) Certifies that the convergence pattern is agreeable. From the sketch it can be perceived that for different combinations of the magnetic field strength $M n$ an appropriate choice of the homotopy parameter $\hbar$ may be selected from the interval $-1<\hbar<-0.5$. Figure (2) demonstrates the convergence loops presented by the nondimensional temperature function $\theta$ schemed against dissimilar values of the Prandtl numbers $\operatorname{Pr}$ plotted for the $20^{\text {th }}$ order approximation of the HAM solutions. From Figure (2) convergence is observed but the interval of convergence reduces with increasing Prandtl numbers Pr. Figure (3) shows the $\hbar$ - curves associated with the nondimensional concentration profile $\psi$ for different values of the Schmidt number $S c$ plotted for the $20^{\text {th }}$ order approximation of the HAM solutions. From Figure (3) a suitable convergence region is obtained.

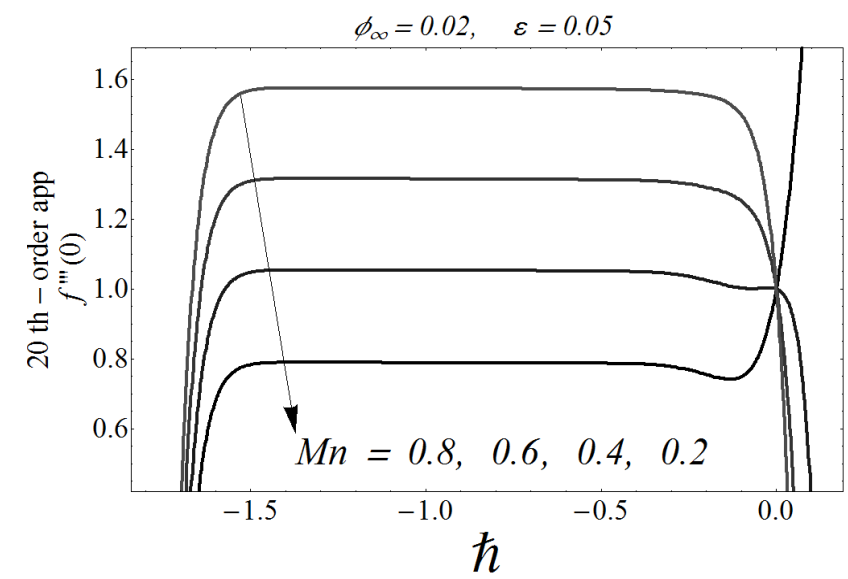

Figure 1. $\hbar$-Curves for the velocity function $f$ against different values of the magnetic parameter $M n$ plotted for the $20^{\text {th }}$ order approximation of the HAM solutions when $k_{1}=0.2, \mathrm{Gr}=0.5, \mathrm{Nr}=0.2, \mathrm{Pr}=0.71, \mathrm{Nr}^{*}=0.2 \mathrm{Nt}$ $=0.5, \mathrm{Nb}=0.25, \mathrm{Sc}=1$.

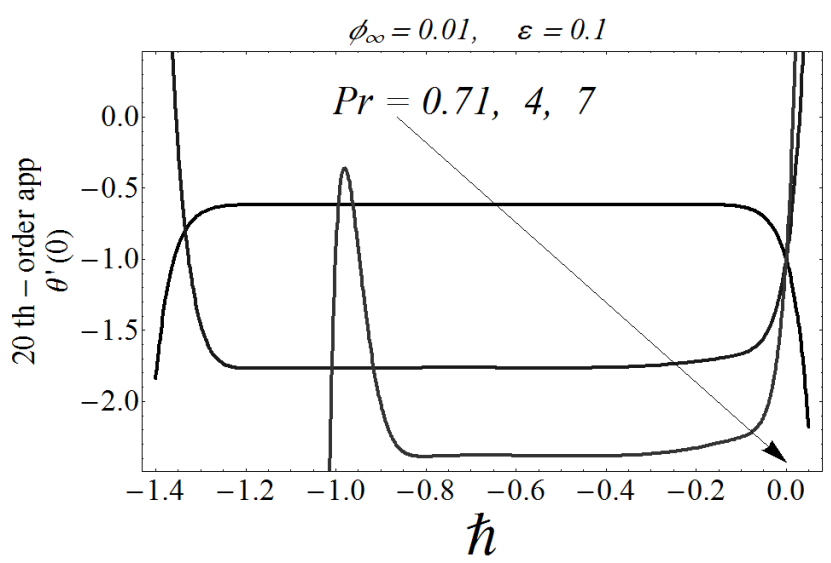

Figure 2. $\hbar$-Curves for the temperature function $\theta$ against different values of the Prandtl number Pr plotted for the $20^{\text {th }}$ order approximation of the HAM solutions when $k_{1}=0.5, \mathrm{Gr}=1, \mathrm{Mn}=0.5, \mathrm{Nr}=0.2, \mathrm{Nr}{ }^{*}=0.2, \mathrm{Nt}$ $=0.5, \mathrm{Nb}=0.25, \mathrm{Sc}=1$. 


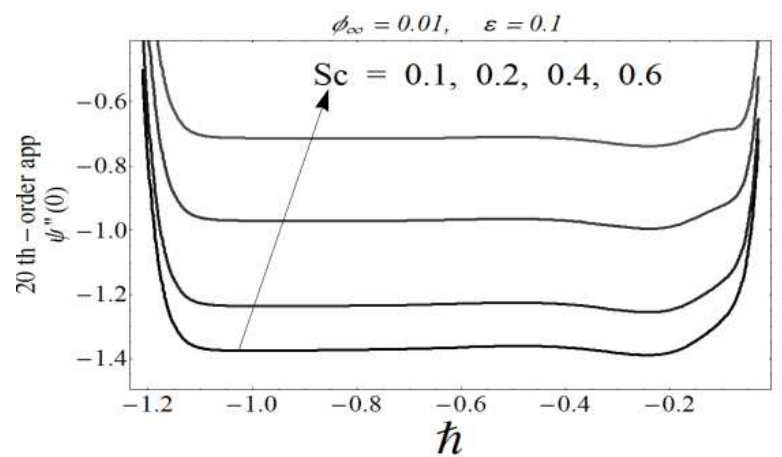

Figure 3. $\hbar$ - Curves for the concentration function $\psi$ against different values of the Schmidt number Sc plotted for the $20^{\text {th }}$ order approximation of the HAM solutions when $k_{1}=0.5, \mathrm{Gr}=1, \mathrm{Mn}=0.5, \mathrm{Nr}=0.2, \mathrm{Pr}=1$, $N r^{*}=0.2, N t=0.5, N b=0.2$.

\section{Results and Discussion}

The governing nonlinear partial differential equations of boundary layer flow of a second grade fluid flowing over a stretching surface such that the fluid contains nanoparticles and is under the action of a uniform magnetic field is studied analytically and the solutions are obtained through the homotopy analysis method. The problem is simplifying under boundary layer assumptions and utilizing a suitable similarity transformation. The impact of different important physical parameters such as the viscoelastic second grade fluid parameter, Prandtl number, Schmidt number, Grashof number, the radiation parameter, the Brownian and the thermophoresis parameters over the nondimensional fluid velocity, heat transfer and the nanoparticle concentration outlines are presented in Figures. (4) to (9). Figure (4) shows the behavior of the nondimensional velocity profile $f^{\prime}(\eta)$ plotted against different combinations of the viscoelastic fluid parameter and the magnetic field parameter $M n$ at the $0.01 \%$ of the nanoparticle concentration keeping the other parameters fixed. From the plot it is noted that by increasing both the magnetic field parameter $M n$ and the viscoelastic second grade fluid parameter the nondimensional velocity profile $f^{\prime}(\eta)$ decreases. The behavior is consistent with the fact that increasing viscoelastic parameter corresponds to an increase in the tensile stress between the fluid layers that in return reduces the fluid velocity. Figure (5) displays the impact of the Grashof number $G r$ and the magnetic field parameter $M n$ over the nondimensional velocity profile $f^{\prime \prime}(\eta)$. From the sketch it is noted that the nondimensional velocity profile $f^{\prime \prime}(\eta)$ increases. The behavior of temperature profile $\theta$ $(\eta)$ for different values of the Prandtl number $P r$ is shown in Figure (6). From the figure a decline in the temperature profile and in the thermal boundary layer is exhibited with an increasing pattern of Prandtl number Pr. Figure (7) offers the manners adopted by the temperature profile $\theta(\eta)$ for different combinations of the thermal radiation parameter $\mathrm{Nr}$ and the Brownian motion parameter $N b$. From the sketch it is evident that the nondimensional temperature profile $\theta(\eta)$ increases with increase in both the Brownian motion parameter $N b$ and the thermal radiation parameter Nr. Figure (8) expresses the influence of the thermophoresis parameter $N t$ and the Prandtl number $\operatorname{Pr}$ over the nanoparticle concentration profile $\psi(\eta)$. From the plot it is noted that an increase in the thermophoresis parameter $N t$ increases the nanoparticle concentration function $\psi(\eta)$. Figure (9) presents the behavior of the Schmidt number $S c$ and the Brownian motion parameter $N b$. From the sketch it is conveyed that increase in the Schmidt number $S c$ decrease the nanoparticle concentration function $\psi(\eta)$. This observation is due to the fact that higher Schmidt numbers corresponds to an enhanced mass transfer rate in the infinite half plane that decreases the concentration at a particular control volume.

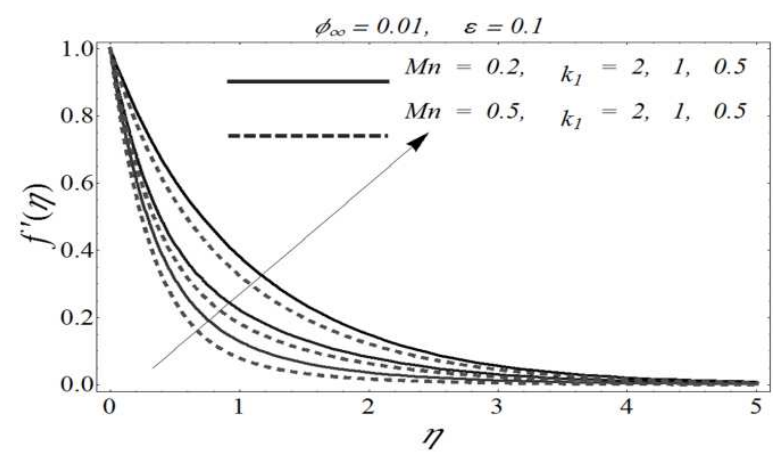

Figure 4. Behavior of velocity profile $f^{\prime}(\eta)$ for different values of the viscoelastic fluid parameter $k_{1}$ and magnetic field parameter $M n$ when $G r$ $=0.5, \mathrm{Nr}=0.2, \mathrm{Pr}=0.71, \mathrm{Nr}^{*}=0.2, \mathrm{Nt}=0.5, \mathrm{Nb}=0.25, \mathrm{Sc}=1$.

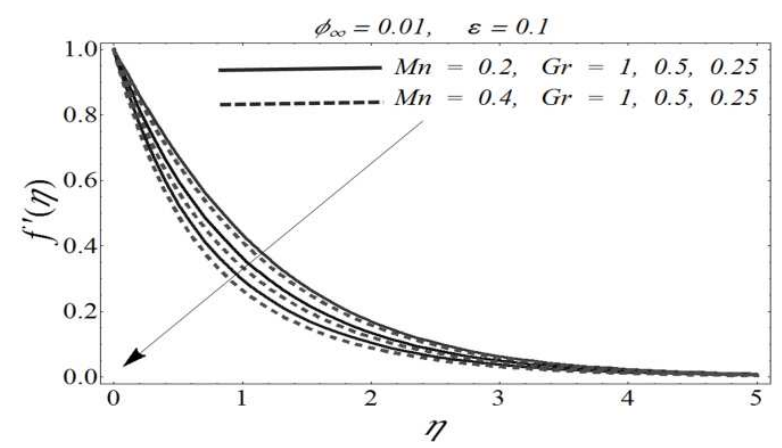

Figure 5. Behavior of velocity profile $f^{\prime}(\eta)$ for different values of the Grashof number $\mathrm{Gr}$ and the magnetic field parameter Mn when $k_{1}=0.5, \mathrm{Nr}=0.2$, $\operatorname{Pr}=1, \mathrm{Nr}^{*}=0.2, \mathrm{Nt}=0.5, \mathrm{Nb}=0.5, \mathrm{Sc}=1$.

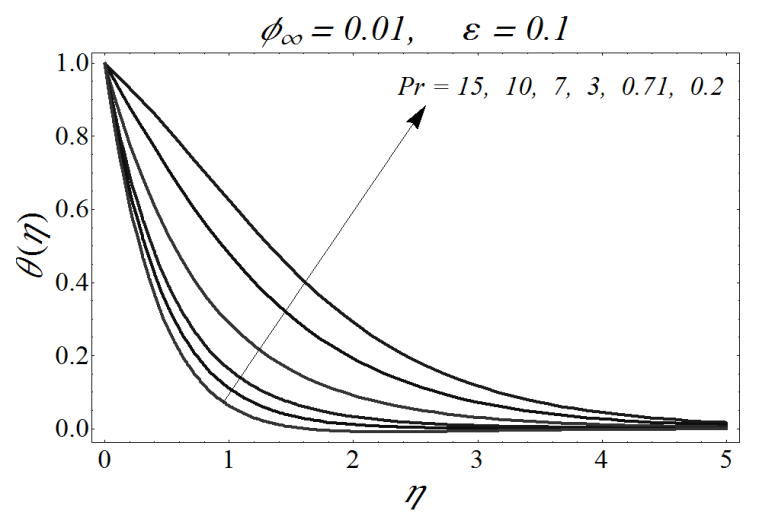

Figure 6. Behavior of temperature profile $\theta(\eta)$ for different values of Prandtl number Pr when $k_{1}=0.5, \mathrm{Nr}=0.2, \mathrm{Gr}=1, \mathrm{Mn}=0.5, \mathrm{Nr}{ }^{*}=0.2$, $N b=0.5, N t=0.5, S c=1$. 


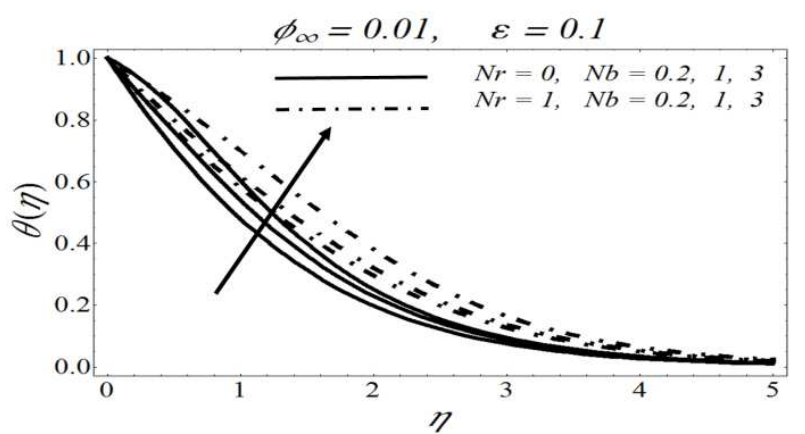

Figure 7. Behavior of temperature profile $\theta$ ( $\eta$ ) for different values of the thermal radiation parameter $\mathrm{Nr}$ and the Brownian motion parameter $\mathrm{Nb}$ when, $k_{1}=0.5, \mathrm{Gr}=1, \mathrm{Mn}=0.5, \mathrm{Pr}=0.7, \mathrm{Nr}^{*}=0.2, \mathrm{Nt}=0.5, \mathrm{Sc}=1$.

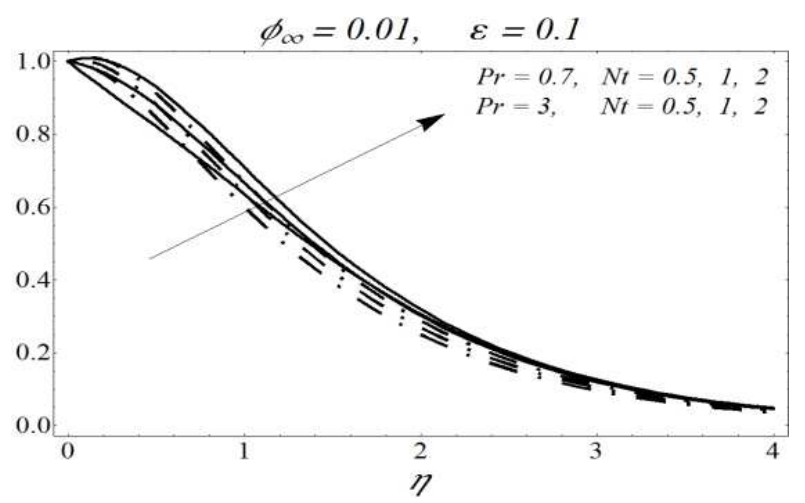

Figure 8. ehavior of nanoparticle concentration profile $\psi(\eta)$ for different values of the Prandtl number Pr and the thermophoresis Nt when, $k_{1}=0.5$, $\mathrm{Gr}=1, \mathrm{Mn}=0.5, \mathrm{Sc}=1, \mathrm{Nr}^{*}=0.2, \mathrm{Nb}=0.2, \mathrm{Nr}=0.2$.

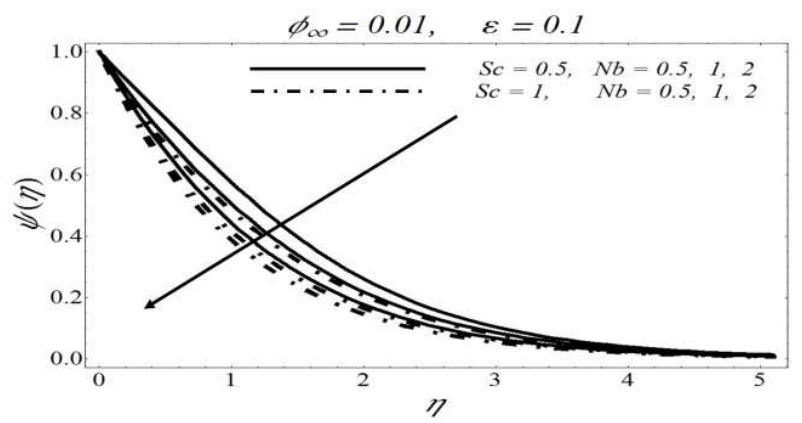

Figure 9. Behavior of nanoparticle concentration profile $\psi(\eta)$ for different values of the Schmidt number $\mathrm{Sc}$ and the Brownian motion parameter $\mathrm{Nb}$ when, $k_{1}=0.5, \mathrm{Gr}=1, \mathrm{Mn}=0.5, \mathrm{Pr}=1, \mathrm{Nr}^{*}=0.2, \mathrm{Nt}=0.5, \mathrm{Nr}=0.5$.

\section{Conclusion}

The problem of steady incompressible boundary layer flow of a non-Newtonian second grade fluid flowing over a linearly stretched sheet is analyzed analytically by means of the homotopy analysis method. The fluid is assumed to be under the influence of a uniform magnetic field. The main findings of the study are listed hare

1. A suitable convergence for the nondimensional velocity, temperature and concentration profiles is achieved through the $\hbar$-curves for a notable choice of the involved parameters.

2. The nondimensional velocity profile is a decreasing function of the viscoelastic and the magnetic parameters while the velocity profile increases by increasing the Grashof number.

3. The nondimensional temperature profile is a decreasing function of the Prandtl numbers.

4. The concentration profile increasing by increasing the thermophoresis parameter, while the concentration function decreases with an increase in the Prandtl number, the Schmidt number and the Brownian motion parameter.

\section{References}

[1] S. U. S. Choi, Enhancing Thermal Conductivity of Fluids With Nanoparticles, Development and Applications of NonNewtonian Flows, in ASME MD- vol. 231 and FED-vol. 66, ed. by DA Siginer, HP Wang (USDOE, Washington, DC (United States), 1995), pp. 99-105.

[2] S. P. Jang, S. U. S. Choi, Effects of various parameters on nanofluid thermal conductivity, J. of Heat Transf. 129 (2007) 617-623.

[3] Md Shakhaoath Khan, Ifsana Karim, Md Sirajul Islam, Mohammad Wahiduzzaman, MHD boundary layer radiative, heat generating and chemical reacting flow past a wedge moving in a nanofluid, Nano Convergence 2014, 1:20.

[4] S. Nadeem, Abdul Rehman, Axisymmetric stagnation flow of a nanofluid in a moving cylinder, Computational Mathematics and Modeling, 24 (2) (2013) 293-306.

[5] Abdul Rehman, S. Nadeem, M. Y. Malik, Stagnation flow of couple stress nanofluid over an exponentially stretching sheet through a porous medium, Journal of Power Technologies 93 (2) (2013) 122-132.

[6] M. Ferdows, Md. Shakhaoath Khan, Md. Mahmud Alam, Shuyu Sun, MHD mixed convective boundary layer flow of a nanofluid through a porous medium due to an exponentially stretching sheet, Mathematical Problems in Engineering, doi:10.1155/2012/408528.

[7] Md Shakhaoath Khan, Ifsana Karim, Lasker Ershad Ali, Ariful Islam, Unsteady MHD free convection boundary-layer flow of a nanofluid along a stretching sheet with thermal radiation and viscous dissipation effects, International Nano Letters (2012), 2-24.

[8] Abdul Rehman, S. Nadeem, S. Iqbal, Muhammad Y. Malik, M. Naseer, Nanoparticle effect over the boundary layer flow over an exponentially stretching cylinder, Proc IMechE Part $\mathrm{N}$ : J Nano engineering and Nano systems DOI: $10.1177 / 1740349913517872$.

[9] M. Y. Malik, M. Naseer, S. Nadeem, Abdul Rehman, The boundary layer flow of Casson nanofluid over a vertical exponentially stretching cylinder, Appl Nanosci, DOI 10.1007/s13204-013-0267-0.

[10] Mohammad Mehdi Keshtkar, Neda Esmaili, Mohammad Reza Ghazanfari, Effect of heat source/sink on MHD mixed convection boundary layer flow on a vertical surface in a porous medium saturated by a nanofluid with suction or injection, Research Inventy: International Journal of Engineering And Science, 4 (5) (2014) 01-11. 
[11] B. Ghasemi, S. M. Aminossadati, Mixed convection in a liddriven triangular enclosure filled with nanofluids, Int. Comm. Heat Mass Transfer, 37 (2010) 1142-1148.

[12] Abdul Rehman, S. Nadeem, Mixed convection heat transfer in micropolar nanofluid over a vertical slender cylinder, Chin Phys Lett 29 (12) (2012) 124701-8.

[13] E. Abu-Nada, Application of nanofluids for heat transfer enhancement of separated flows encountered in a backward facing step, Int. J. Heat Fluid Flow 29 (2008) 242-249.

[14] M. M. Rashidi, E. Momoniat, B. Rostami, Analytic approximate solutions for MHD boundary-layer viscoelastic fluid flow over continuously moving stretching surface by homotopy analysis method with two auxiliary parameters, Journal of Applied Mathematics, Doi:10.1155/2012/780415.

[15] S. Nadeem, Abdul Rehman, K. Vajravelu, Jinho Lee, Changhoon Lee, Axisymmetric stagnation flow of a micropolar nanofluid in a moving cylinder, Mathematical Problems in Engineering, Volume 2012, Article ID 378259.

[16] S. Nadeem, Abdul Rehman, Changhoon Lee, Jinho Lee, Boundary layer flow of second grade fluid in a cylinder with heat transfer, Mathematical Problems in Engineering, Volume 2012, Article ID 640289.

[17] S. Nadeem, Abdul Rehman, Mohamed Ali, The boundary layer flow and heat transfer of a nanofluid over a vertical slender cylinder, J. Nano Engineering and Nano Systems (2012) 1-9.

[18] Abdul Rehman, S. Nadeem, M. Y. Malik, Boundary layer stagnation-point flow of a third grade fluid over an exponentially stretching sheet, Braz. J. Che. Eng. 30 (3) (2013) 611-618.

[19] Abdul Rehman, S. Nadeem, Heat transfer analysis of the boundary layer flow over a vertical exponentially stretching cylinder, Global J. Sci. Fron. Res. 13 (11) (2013) 73-85.

[20] M. Y. Malik, M. Naseer, S. Nadeem, Abdul Rehman, The boundary layer flow of hyperbolic tangent fluid over a vertical exponentially stretching cylinder, Alexandria Engineering J., 53 (2014) 747-750.

[21] M. Y. Malik, M. Naseer, Abdul Rehman, Numerical study of convective heat transfer on the Power Law fluid over a vertical exponentially stretching cylinder, Applied and Computational Mathematics, 4 (5), (2015) 346-350.

[22] Abdul Rehman, Razmak Bazai, Sallahuddin Achakzai, Saleem Iqbal, Muhammad Naseer, Boundary Layer Flow and Heat Transfer of Micropolar Fluid over a Vertical Exponentially Stretched Cylinder, Applied and Computational Mathematics, 4 (6) (2015) 424-430.

[23] Abdul Rehman, Ghulam Farooq, Israr Ahmed, Muhammad Naseer, Muhammad Zulfiqar, Boundary Layer StagnationPoint Flow of Second Grade Fluid over an Exponentially Stretching Sheet, American Journal of Applied Mathematics and Statistics, 3 (6) (2015) 211-219.

[24] Abdul Rehmana, Sallahuddin Achakzai, Sohail Nadeem, Saleem Iqbal, Stagnation point flow of Eyring Powell fluid in a vertical cylinder with heat transfer, Journal of Power Technologies 96 (1) (2016) 57-62.

[25] Abdul Rehman, Saleem Iqbal, Syed Mohsin Raza, Axisymmetric Stagnation Flow of a Micropolar Fluid in a Moving Cylinder: An Analytical Solution, Fluid Mechanics, 2 (1) (2016) 1-7.

[26] Abdul Rehman, Naveed Sheikh, Boundary Layer StagnationPoint Flow of Micropolar Fluid over an Exponentially Stretching Sheet, International Journal of Fluid Mechanics \& Thermal Sciences, 3 (3) (2017) 25-31. 